\title{
Berry-Esséen bound of sample quantiles for negatively associated sequence
}

\author{
Wenzhi Yang ${ }^{1}$, Shuhe $\mathrm{Hu}^{1 *}$, Xuejun Wang ${ }^{1}$ and Qinchi Zhang ${ }^{2}$
}

\author{
* Correspondence: hushuhe@263. \\ net \\ ${ }^{1}$ School of Mathematical Science, \\ Anhui University Hefei 230039, PR \\ China \\ Full list of author information is \\ available at the end of the article
}

\section{Abstract}

In this paper, we investigate the Berry-Esséen bound of the sample quantiles for the negatively associated random variables under some weak conditions. The rate of normal approximation is shown as $O\left(n^{-1 / 9}\right)$.

2010 Mathematics Subject Classification: 62F12; 62E20; 60F05.

Keywords: Berry-Ess?é?en bound, sample quantile, negatively associated

\section{Introduction}

Assume that $\left\{X_{n}\right\}_{n \geq 1}$ is a sequence of random variables defined on a fixed probability space $(\Omega, \mathcal{F}, P)$ with a common marginal distribution function $F(x)=P\left(X_{1} \leq x\right) . F$ is a distribution function (continuous from the right, as usual). For $0<p<1$, the $p$ th quantile of $F$ is defined as

$$
\xi_{p}=\inf \{x: F(x) \geq p\}
$$

and is alternately denoted by $F^{-1}(p)$. The function $F^{-1}(t), 0<t<1$, is called the inverse function of $F$. It is easy to check that $\xi_{p}$ possesses the following properties:

(i) $F\left(\xi_{p^{-}}\right) \leq p \leq F\left(\xi_{p}\right)$;

(ii) if $\xi_{p}$ is the unique solution $x$ of $F(x-) \leq p \leq F(x)$, then for any $\varepsilon>0$,

$$
F\left(\xi_{p}-\varepsilon\right)<p<F\left(\xi_{p}+\varepsilon\right) .
$$

For a sample $X_{1}, X_{2}, \ldots, X_{n}, n \geq 1$, let $F_{n}$ represent the empirical distribution function based on $X_{1}, X_{2}, \ldots, X_{n}$, which is defined as $F_{n}(x)=\frac{1}{n} \sum_{i=1}^{n} I\left(X_{i} \leq x\right), x \in \mathbb{R}$, where $I(A)$ denotes the indicator function of a set $A$ and $\mathbb{R}$ is the real line. For $0<p<1$, we define $F_{n}^{-1}(p)=\inf \left\{x: F_{n}(x) \geq p\right\}$ as the $p$ th quantile of sample.

Recall that a finite family $\left\{X_{1}, \ldots, X_{n}\right\}$ is said to be negatively associated (NA) if for any disjoint subsets $A, B \subset\{1,2, \ldots, n\}$, and any real coordinatewise nondecreasing functions $f$ on $R^{A}, g$ on $R^{B}$,

$$
\operatorname{Cov}\left(f\left(X_{k}, k \in A\right), g\left(X_{k}, k \in B\right)\right) \leq 0 .
$$

A sequence of random variables $\left\{X_{i}\right\}_{i \geq 1}$ is said to be NA if for every $n \geq 2, X_{1}, X_{2}, \ldots$, $X_{n}$ are NA.

From 1960s, many authors have obtained the asymptotic results for the sample quantiles, including the well-known Bahadur representation. Bahadur [1] firstly introduced

(c) 2011 Yang et al; licensee Springer. This is an Open Access article distributed under the terms of the Creative Commons Attribution License (http://creativecommons.org/licenses/by/2.0), which permits unrestricted use, distribution, and reproduction in any medium, provided the original work is properly cited. 
an elegant representation for the sample quantiles in terms of empirical distribution function based on independent and identically distributed (i.i.d.) random variables. Sen [2], Babu and Singh [3] and Yoshihara [4] gave the Bahadur representation for the sample quantiles under $\varphi$-mixing sequence and $\alpha$-mixing sequence, respectively. Sun [5] established the Bahadur representation for the sample quantiles under $\alpha$-mixing sequence with polynomially decaying rate. Ling [6] investigated the Bahadur representation for the sample quantiles under NA sequence. Li et al. [7] investigated the Bahadur representation of the sample quantile based on negatively orthant-dependent (NOD) sequence, which is weaker than NA sequence. Xing and Yang [8] also studied the Bahadur representation for the sample quantiles under NA sequence. Wang et al. [9] revised the results of Sun [5] and got a better bound. For more details about Bahadur representation, one can refer to Serfling [10].

For a fixed $p \in(0,1)$, let $\xi_{p}=F^{-1}(p), \xi_{p, n}=F_{n}^{-1}(p)$ and $\Phi(t)$ be the distribution function of a standard normal variable. In [[10], p. 81], the Berry-Esséen bound of the sample quantiles for i.i.d. random variables is given as follows:

Theorem A Let $0<p<1$ and $\left\{X_{n}\right\}_{n \geq 1}$ be a sequence of i.i.d. random variables. Suppose that in a neighborhood of $\xi_{p}$, F possesses a positive continuous density $f$ and a bounded second derivative $F^{\prime \prime}$. Then

$$
\sup _{-\infty<t<\infty}\left|P\left(\frac{n^{1 / 2}\left(\xi_{p, n}-\xi_{p}\right)}{[p(1-p)]^{1 / 2} / f\left(\xi_{p}\right)} \leq t\right)-\Phi(t)\right|=O\left(n^{-1 / 2}\right), \quad n \rightarrow \infty .
$$

In this paper, we investigate the Berry-Esséen bound of the sample quantiles for NA random variables under some weak conditions. The rate of normal approximation is shown as $O\left(n^{-1 / 9}\right)$.

Berry-Esséen theorem, which is known as the rate of convergence in the central limit theorem, can be found in many monographs such as Shiryaev [11], Petrov [12]. For the case of i.i.d. random variables, the optimal rate is $O\left(n^{-\frac{1}{2}}\right)$, and for the case of martingale, the rate is $O\left(n^{-\frac{1}{4}} \log n\right)$ [[13], Chapter 3]. For other papers about Berry-Esséen bound, for example, under the association sample, Cai and Roussas [14,15] studied the Berry-Esséen bounds for the smooth estimator of quantiles and the smooth estimator of a distribution function, respectively; Yang [16] obtained the Berry-Esséen bound of the regression weighted estimator for NA sequence; Wang and Zhang [17] provided the Berry-Esséen bound for linear negative quadrant-dependent (LNQD) sequence; Liang and Baek [18] gave the Berry-Esséen bounds for density estimates under NA sequence; Liang and Uña-Álvarez [19] studied the Berry-Esséen bound in kernel density estimation for $\alpha$-mixing censored sample; Lahiri and Sun [20] obtained the BerryEsséen bound of the sample quantiles for $\alpha$-mixing random variables, etc.

Throughout the paper, $C, C_{1}, C_{2}, C_{3}, \ldots, d$ denote some positive constants not depending on $n$, which may be different in various places. $\lfloor x\rfloor$ denotes the largest integer not exceeding $x$, and the second-order stationarity means that

$$
\left(X_{1}, X_{1+k}\right) \stackrel{d}{=}\left(X_{i}, X_{i+k}\right), \quad i \geq 1, k \geq 1 .
$$

Inspired by Serfling [10], Cai and Roussas [14,15], Yang [16], Liang and Uña-Álvarez [19], Lahiri and Sun [20], etc., we obtain Theorem 1.1 in Section 1. Two preliminary 
lemmas are given in Section 2, and the proof of Theorem 1.1 is given in Section 3. Next, we give the main result as follows:

Theorem 1.1 Let $0<p<1$ and $\left\{X_{n}\right\}_{n \geq 1}$ be a second-order stationary NA sequence with common marginal distribution function $F$ and $E X_{n}=0$ for $n=1,2, \ldots$ Assume that in a neighborhood of $\xi_{p}, F$ possesses a positive continuous density $f$ and a bounded second derivative $F^{\prime \prime}$. If there exists an $\varepsilon_{0}>0$ such that for $x \in\left[\xi_{p}-\varepsilon_{0}, \xi_{p}+\varepsilon_{0}\right]$,

$$
\sum_{j=2}^{\infty} j\left|\operatorname{Cov}\left[I\left(X_{1} \leq x\right), I\left(X_{j} \leq x\right)\right]\right|<\infty,
$$

and

$$
\operatorname{Var}\left[I\left(X_{1} \leq \xi_{p}\right)\right]+2 \sum_{j=2}^{\infty} \operatorname{Cov}\left[I\left(X_{1} \leq \xi_{p}\right), I\left(X_{j} \leq \xi_{p}\right)\right]:=\sigma^{2}\left(\xi_{p}\right)>0,
$$

then

$$
\sup _{-\infty<t<\infty}\left|P\left(\frac{n^{1 / 2}\left(\xi_{p, n}-\xi_{p}\right)}{\sigma\left(\xi_{p}\right) / f\left(\xi_{p}\right)} \leq t\right)-\Phi(t)\right|=O\left(n^{-1 / 9}\right), \quad n \rightarrow \infty .
$$

Remark 1.1 Assumption (1.2) is a general condition, see for example Cai and Roussas [14]. For the stationary sequences of associated and negatively associated, Cai and Roussas [15] gave the notation $\mu(n)=\sum_{j=n}^{\infty}\left|\operatorname{Cov}\left(X_{1}, X_{j+1}\right)\right|^{1 / 3}$ and supposed that $\mu(1)<$ $\infty$. In addition, they supposed that $\mu(n)=O\left(n^{-\alpha}\right)$ for some $\alpha>0$ or $\delta(1)<\infty$, where $\delta(i)=\sum_{j=i}^{\infty} \mu(j)$, then obtained the Berry-Esséen bounds for smooth estimator of a distribution function. Under the assumptions $\sum_{j=n+1}^{\infty}\left\{\operatorname{Cov}\left(X_{1}, X_{j}\right)\right\}^{1 / 3}=O\left(n^{-(r-1)}\right)$ for some $r>1$ or $\sum_{n=1}^{\infty} \mathrm{n}^{7} \operatorname{Cov}\left(X_{1}, X_{n}\right)<\infty$, Chaubey et al. [21] studied the smooth estimation of survival and density functions for a stationary-associated process using Poisson weights. In this paper, for $x \in\left[\xi_{p}-\varepsilon_{0}, \xi_{p}+\varepsilon_{0}\right]$, the assumption (1.1) has some restriction on the covariances of $\operatorname{Cov}\left[I\left(X_{1} \leq x\right), I\left(X_{j} \leq x\right)\right]$ in the neighborhood of $\xi_{p}$.

\section{Preliminaries}

Lemma 2.1 Let $\left\{X_{n}\right\}_{n \geq 1}$ be a stationary NA sequence with $E X_{n}=0,\left|X_{n}\right| \leq d<\infty$ for $n=$ $1,2, \ldots$. There exists some $\beta \geq 1$ such that $\sum_{j=b_{n}}^{\infty}\left|\operatorname{Cov}\left(X_{1}, X_{j}\right)\right|=O\left(b_{n}^{-\beta}\right)$ for all $0<b_{n} \rightarrow$ $\infty$ as $n \rightarrow \infty$. If

$$
\liminf _{n \rightarrow \infty} n^{-1} \operatorname{Var}\left(\sum_{i=1}^{n} X_{i}\right)=\sigma_{0}^{2}>0,
$$

then

$$
\sup _{-\infty<t<\infty}\left|P\left(\frac{\sum_{i=1}^{n} X_{i}}{\sqrt{\operatorname{Var}\left(\sum_{i=1}^{n} X_{i}\right)}} \leq t\right)-\Phi(t)\right|=O\left(n^{-1 / 9}\right), \quad n \rightarrow \infty .
$$

Proof We employ Bernstein's big-block and small-block procedure. Partition the set $\{1,2, \ldots, n\}$ into $2 k_{n}+1$ subsets with large blocks of size $\mu=\mu_{n}$ and small block of size $v=v_{n}$. Define

$$
\mu_{n}=\left[n^{2 / 3}\right], v_{n}=\left[n^{1 / 3}\right], \quad k=k_{n}:=\left\lfloor\frac{n}{\mu_{n}+v_{n}}\right\rfloor=\left[n^{1 / 3}\right],
$$


and $Z_{n, i}=X_{i} / \sqrt{\operatorname{Var}\left(\sum_{i=1}^{n} X_{i}\right)}$. Let $\eta_{j}, \xi_{j}, \zeta_{j}$ be defined as follows:

$$
\begin{aligned}
& \eta_{j}:=\sum_{i=j(\mu+v)+1}^{j(\mu+v)+\mu} Z_{n, i}, \quad 0 \leq j \leq k-1, \\
& \xi_{j}:=\sum_{i=j(\mu+v)+\mu+1}^{(j+1)(\mu+\nu)} Z_{n, i}, \quad 0 \leq j \leq k-1, \\
& \zeta_{k}:=\sum_{i=k(\mu+v)+1}^{n} Z_{n, i} .
\end{aligned}
$$

Write

$$
S_{n}:=\frac{\sum_{i=1}^{n} X_{i}}{\sqrt{\operatorname{Var}\left(\sum_{i=1}^{n} X_{i}\right)}}=\sum_{j=0}^{k-1} \eta_{j}+\sum_{j=0}^{k-1} \xi_{j}+\zeta_{k}:=S_{n}^{\prime}+S_{n}^{\prime \prime}+S_{n}^{\prime \prime \prime} .
$$

By Lemma A.3, we can see that

$$
\begin{aligned}
& \sup _{-\infty<t<\infty}\left|P\left(S_{n} \leq t\right)-\Phi(t)\right| \\
= & \sup _{-\infty<t<\infty}\left|P\left(S_{n}^{\prime}{ }_{n}+S^{\prime \prime}{ }_{n}+S^{\prime \prime \prime}{ }_{n} \leq t\right)-\Phi(t)\right| \leq \sup _{-\infty<t<\infty}\left|P\left(S_{n}^{\prime} \leq t\right)-\Phi(t)\right| \\
& +\frac{2 n^{-\frac{1}{9}}}{\sqrt{2 \pi}}+P\left(\left|S^{\prime \prime}{ }_{n}\right|>n^{-\frac{1}{9}}\right)+P\left(\left|S^{\prime \prime \prime}{ }_{n}\right|>n^{-\frac{1}{9}}\right) .
\end{aligned}
$$

Firstly, we estimate $E\left(S_{n}^{\prime \prime}\right)^{2}$ and $E\left(S_{n}^{\prime \prime \prime}\right)^{2}$, which will be used to estimate $P\left(\left|S_{n}^{\prime \prime}\right|>n^{-\frac{1}{9}}\right)$ and $P\left(\left|S_{n}^{\prime \prime \prime}\right|>n^{-\frac{1}{9}}\right)$ in (2.7). By the conditions $\left|X_{i}\right| \leq d$ and $\lim _{n \rightarrow \infty} \inf n^{-1} \operatorname{Var}\left(\sum_{i=1}^{n} X_{i}\right)=\sigma_{0}^{2}>0$, it is easy to see that $\left|Z_{n, i}\right| \leq \frac{C_{1}}{\sqrt{n}}$. And $E\left(\xi_{j}\right)^{2} \leq C v_{n} \mid$ $n$ follows from $E Z_{n, i}=0$ and Lemma A.1. Combining the definition of NA with the definition of $\xi_{j}, j=0,1, \ldots, k-1$, we can easily prove that $\left\{\xi_{0}, \xi_{1}, \ldots, \xi_{k-1}\right\}$ is NA. Therefore, it follows from (2.2), (2.4), (2.6) and Lemma A.1 that

$$
E\left(S_{n}^{\prime \prime}\right)^{2} \leq C_{1} \sum_{j=0}^{k-1} E \xi_{j}^{2} \leq C_{2} \frac{k_{n} v_{n}}{n} \leq C_{3} \frac{n}{\mu_{n}+v_{n}} \frac{v_{n}}{n} \leq C_{4} \frac{v_{n}}{\mu_{n}}=O\left(n^{-1 / 3}\right) .
$$

On the other hand, we can get that

$$
\begin{aligned}
E\left(S_{n}^{\prime \prime \prime}\right)^{2} & \leq \frac{C_{5}}{n} E\left(\sum_{i=k(\mu+v)+1}^{n} X_{i}\right)^{2} \leq \frac{C_{6}}{n} \sum_{i=k(\mu+\nu)+1}^{n} E X_{i}^{2} \\
& \leq \frac{C_{7}}{n}\left(n-k_{n}\left(\mu_{n}+v_{n}\right)\right) \leq C_{8} \frac{\mu_{n}+v_{n}}{n}=O\left(n^{-1 / 3}\right)
\end{aligned}
$$

from (2.5), $\liminf _{n \rightarrow \infty} n^{-1} \operatorname{Var}\left(\sum_{i=1}^{n} X_{i}\right)=\sigma_{0}^{2}>0,\left|X_{i}\right| \leq d$ and Lemma A.1. Consequently, by Markov's inequality, (2.8) and (2.9),

$$
P\left(\left|S^{\prime \prime}{ }_{n}\right|>n^{-\frac{1}{9}}\right) \leq n^{\frac{2}{9}} \cdot E\left(S_{n}^{\prime \prime}\right)^{2}=O\left(n^{-1 / 9}\right),
$$




$$
P\left(\left|S^{\prime \prime \prime}{ }_{n}\right|>n^{-\frac{1}{9}}\right) \leq n^{\frac{2}{9}} \cdot E\left(S_{n}^{\prime \prime \prime}\right)^{2}=O\left(n^{-1 / 9}\right) .
$$

In the following, we will estimate $\sup _{-\infty<t<\infty}\left|P\left(S_{n}^{\prime} \leq t\right)-\Phi(t)\right|$. Define

$$
s_{n}^{2}:=\sum_{j=0}^{k-1} \operatorname{Var}\left(\eta_{j}\right), \quad \Gamma_{n}:=\sum_{0 \leq i<j \leq k-1} \operatorname{Cov}\left(\eta_{i}, \eta_{j}\right) .
$$

Here, we first estimate the growth rate $\left|s_{n}^{2}-1\right|$. Since $E S_{n}^{2}=1$ and

$$
E\left(S_{n}^{\prime}\right)^{2}=E\left[S_{n}-\left(S_{n}^{\prime \prime}+S_{n}^{\prime \prime \prime}\right)\right]^{2}=1+E\left(S_{n}^{\prime \prime}+S_{n}^{\prime \prime \prime}\right)^{2}-2 E\left[S_{n}\left(S_{n}^{\prime \prime}+S_{n}^{\prime \prime \prime}\right)\right],
$$

by (2.8) and (2.9), it has

$$
\begin{aligned}
\left|E\left(S_{n}^{\prime}\right)^{2}-1\right|= & \left|E\left(S_{n}^{\prime \prime}+S_{n}^{\prime \prime \prime}\right)^{2}-2 E\left[S_{n}\left(S_{n}^{\prime \prime}+S_{n}^{\prime \prime \prime}\right)\right]\right| \\
\leq & E\left(S_{n}^{\prime \prime}\right)^{2}+E\left(S_{n}^{\prime \prime \prime}\right)^{2}+2\left[E\left(S_{n}^{\prime \prime}\right)^{2}\right]^{1 / 2}\left[E\left(S_{n}^{\prime \prime \prime}\right)^{2}\right]^{1 / 2} \\
& +2\left[E\left(S_{n}^{2}\right)\right]^{1 / 2}\left[E\left(S_{n}^{\prime \prime}\right)^{2}\right]^{1 / 2}+2\left[E\left(S_{n}^{2}\right)\right]^{1 / 2}\left[E\left(S_{n}^{\prime \prime \prime}\right)^{2}\right]^{1 / 2} \\
= & O\left(n^{-1 / 3}\right)+O\left(n^{-1 / 6}\right)=O\left(n^{-1 / 6}\right) .
\end{aligned}
$$

Notice that

$$
s_{n}^{2}=E\left(S_{n}^{\prime}\right)^{2}-2 \Gamma_{n} .
$$

With $\lambda_{j}=j\left(\mu_{n}+v_{n}\right)$,

$$
2 \Gamma_{n}=2 \sum_{0 \leq i<j \leq k-1} \sum_{l_{1}=1}^{\mu_{n}} \sum_{l_{2}=1}^{\mu_{n}} \operatorname{Cov}\left(Z_{n, \lambda_{i}+l_{1}}, Z_{n, \lambda_{j}+l_{2}}\right),
$$

but since $i \neq j,\left|\lambda_{i}-\lambda_{j}+l_{1}-l_{2}\right| \geq v_{n}$, it has that

$$
\begin{aligned}
\left|2 \Gamma_{n}\right| & \leq 2 \sum_{\substack{1 \leq i<j \leq n \\
j-i \geq v_{n}}}\left|\operatorname{Cov}\left(Z_{n, i}, Z_{n, j}\right)\right| \leq \frac{C_{1}}{n} \sum_{\substack{1 \leq i<j \leq n \\
j-i \geq v_{n}}}\left|\operatorname{Cov}\left(X_{i}, X_{j}\right)\right| \\
& \leq C_{2} \sum_{k \geq v_{n}}\left|\operatorname{Cov}\left(X_{1}, X_{k}\right)\right|=O\left(n^{-\beta / 3}\right)=O\left(n^{-1 / 3}\right)
\end{aligned}
$$

following from (2.2) and the conditions of stationary, $\liminf _{n \rightarrow \infty} n^{-1} \operatorname{Var}\left(\sum_{i=1}^{n} X_{i}\right)=\sigma_{0}^{2}>0$ and $\sum_{j=b_{n}}^{\infty}\left|\operatorname{Cov}\left(X_{1}, X_{j}\right)\right|=O\left(b_{n}^{-\beta}\right), \beta \geq 1$. So, by (2.12), (2.13) and (2.14), we can get that

$$
\left|s_{n}^{2}-1\right|=O\left(n^{-1 / 6}\right)+O\left(n^{-1 / 3}\right)=O\left(n^{-1 / 6}\right) .
$$

For $j=0,1, \ldots, k-1$, let $\eta_{j}^{\prime}$ be the independent random variables and $\left|s_{n}^{2}-1\right|=O\left(n^{-1 / 6}\right)+O\left(n^{-1 / 3}\right)=O\left(n^{-1 / 6}\right)$. have the same distribution as $\eta_{j}, j=0,1, \ldots$, $k-1$. Define $H_{n}=\sum_{j=0}^{k-1} \eta^{\prime}$. It can be found that

$$
\begin{aligned}
& \sup _{-\infty<t<\infty}\left|P\left(S_{n}^{\prime} \leq t\right)-\Phi(t)\right| \\
\leq & \sup _{-\infty<t<\infty}\left|P\left(S_{n}^{\prime} \leq t\right)-P\left(H_{n} \leq t\right)\right|+\sup _{-\infty<t<\infty}\left|P\left(H_{n} \leq t\right)-\Phi\left(t / s_{n}\right)\right| \\
& +\sup _{-\infty<t<\infty}\left|\Phi\left(t / s_{n}\right)-\Phi(t)\right|:=D_{1}+D_{2}+D_{3} .
\end{aligned}
$$

Let $\varphi(t)$ and $\psi(t)$ be the characteristic functions of $S_{n}^{\prime}$ and $H_{n}$, respectively. By Esséen inequality [[12], Theorem 5.3], for any $T>0$, 


$$
\begin{aligned}
D_{1} & \leq \int_{-T}^{T}\left|\frac{\phi(t)-\psi(t)}{t}\right| d t+T \sup _{-\infty<t<\infty} \int_{|u| \leq \frac{C}{T}}\left|P\left(H_{n} \leq u+t\right)-P\left(H_{n} \leq t\right)\right| \mathrm{d} u \\
& :=D_{1 n}+D_{2 n} .
\end{aligned}
$$

With $\lambda_{j}=j\left(\mu_{n}+v_{n}\right)$ and similar to the proof of Lemma 3.4 of Yang [16], we have that

$$
\begin{aligned}
|\varphi(t)-\psi(t)| & =\left|E \exp \left(i t \sum_{j=0}^{k-1} \eta_{j}\right)-\prod_{j=0}^{k-1} E \exp \left(i t \eta_{j}\right)\right| \\
& \leq 4 t^{2} \sum_{\substack{0 \leq i<j \leq k-1 \\
\mu_{1}=1}} \sum_{l_{2}=1}^{\mu_{n}}\left|\operatorname{Cov}\left(Z_{n, \lambda_{i}+l_{1}}, Z_{n, \lambda_{j}+l_{2}}\right)\right| \\
& \leq \frac{C_{1} t^{2}}{n} \sum_{\substack{1 \leq i<j \leq n \\
j-i \geq v_{n}}}\left|\operatorname{Cov}\left(X_{i}, X_{j}\right)\right| \\
& \leq C_{2} t^{2} \sum_{j \geq v_{n}}\left|\operatorname{Cov}\left(X_{1}, X_{j}\right)\right| \leq C_{3} t^{2} n^{-\beta / 3}
\end{aligned}
$$

by (2.2) and the conditions of stationary, $\liminf _{n \rightarrow \infty} n^{-1} \operatorname{Var}\left(\sum_{i=1}^{n} X_{i}\right)=\sigma_{0}^{2}>0$ and $\sum_{j=b_{n}}^{\infty}\left|\operatorname{Cov}\left(X_{1}, X_{j}\right)\right|=O\left(b_{n}^{-\beta}\right)$. Set $T=n^{(3 \beta-1) / 18}$ for $\beta \geq 1$, we have by (2.18) that

$$
D_{1 n}=\int_{-T}^{T}\left|\frac{\varphi(t)-\psi(t)}{t}\right| d t \leq C n^{-\beta / 3} \cdot T^{2}=O\left(n^{-1 / 9}\right) .
$$

It follows from the Berry-Esséen inequality [[12], Theorem 5.7], that

$$
\sup _{-\infty<t<\infty}\left|P\left(H_{n} / s_{n} \leq t\right)-\Phi(t)\right| \leq \frac{C}{s_{n}^{3}} \sum_{j=0}^{k-1} E\left|\eta_{j}^{\prime}\right|^{3}=\frac{C}{s_{n}^{3}} \sum_{j=0}^{k-1} E\left|\eta_{j}\right|^{3} .
$$

By (2.3) and Lemma A.1,

$$
\begin{aligned}
& \sum_{j=0}^{k-1} E\left|\eta_{j}\right|^{3}=\sum_{j=0}^{k-1} E\left|\sum_{i=j(\mu+\nu)+1}^{j(\mu+v)+\mu} Z_{n, i}\right|^{3} \\
\leq & \frac{C_{1}}{n^{3 / 2}} \sum_{j=0}^{k-1} E\left|\sum_{i=j(\mu+\nu)+1}^{j(\mu+v)+\mu} X_{i}\right|^{3} \\
\leq & \frac{C_{2}}{n^{3 / 2}} \sum_{j=0}^{k-1}\left\{\sum_{i=j(\mu+\nu)+1}^{j(\mu+\nu)+\mu} E\left|X_{i}\right|^{3}+\left(\sum_{i=j(\mu+\nu)+1}^{j(\mu+\nu)+\mu} E\left|X_{i}\right|^{2}\right)^{3 / 2}\right\} \\
\leq & \frac{C_{3}}{n^{3 / 2}} \sum_{j=0}^{k-1}\left(\mu+\mu^{3 / 2}\right) \leq \frac{C_{4} k \mu^{3 / 2}}{n^{3 / 2}}=O\left(n^{-1 / 6}\right) .
\end{aligned}
$$

Combining (2.20) with (2.21), we obtain that

$$
\sup _{-\infty<t<\infty}\left|P\left(\frac{H_{n}}{s_{n}} \leq t\right)-\Phi(t)\right|=O\left(n^{-1 / 6}\right),
$$


since $s_{n} \rightarrow 1$ as $n \rightarrow \infty$ by (2.15). It follows from (2.22) that

$$
\begin{aligned}
& \sup _{-\infty<t<\infty}\left|P\left(H_{n} \leq u+t\right)-P\left(H_{n} \leq t\right)\right| \\
\leq & \sup _{-\infty<t<\infty}\left|P\left(\frac{H_{n}}{s_{n}} \leq \frac{u+t}{s_{n}}\right)-\Phi\left(\frac{u+t}{s_{n}}\right)\right| \\
& +\sup _{-\infty<t<\infty}\left|P\left(\frac{H_{n}}{s_{n}} \leq \frac{t}{s_{n}}\right)-\Phi\left(\frac{t}{s_{n}}\right)\right|+\sup _{-\infty<t<\infty}\left|\Phi\left(\frac{u+t}{s_{n}}\right)-\Phi\left(\frac{t}{s_{n}}\right)\right| \\
\leq & 2 \sup _{-\infty<t<\infty}\left|P\left(\frac{H_{n}}{s_{n}} \leq t\right)-\Phi(t)\right|+\sup _{-\infty<t<\infty}\left|\Phi\left(\frac{u+t}{s_{n}}\right)-\Phi\left(\frac{t}{s_{n}}\right)\right| \\
= & O\left(n^{-1 / 6}\right)+O\left(\frac{|u|}{s_{n}}\right),
\end{aligned}
$$

which implies that

$$
\begin{aligned}
D_{2 n} & =T \sup _{-\infty<t<\infty} \int_{|u| \leq C / T}\left|P\left(H_{n} \leq u+t\right)-P\left(H_{n} \leq t\right)\right| \mathrm{d} u \\
& \leq \frac{C_{1}}{n^{1 / 6}}+\frac{C_{2}}{T}=O\left(n^{-1 / 6}\right)+O\left(n^{-1 / 9}\right)=O\left(n^{-1 / 9}\right),
\end{aligned}
$$

where $T=n^{(3 \beta-1) / 18}$. It is known that [[12], Lemma 5.2],

$$
\sup _{-\infty<x<\infty}|\Phi(p x)-\Phi(x)| \leq \frac{(p-1) I(p \geq 1)}{(2 \pi e)^{1 / 2}}+\frac{\left(p^{-1}-1\right) I(0<p<1)}{(2 \pi e)^{1 / 2}} .
$$

Thus, by (2.15),

$$
\begin{aligned}
D_{3} & =\sup _{-\infty<t<\infty}\left|\Phi\left(t / s_{n}\right)-\Phi(t)\right| \\
& \leq(2 \pi e)^{-1 / 2}\left(s_{n}-1\right) I\left(s_{n} \geq 1\right)+(2 \pi e)^{-1 / 2}\left(s_{n}^{-1}-1\right) I\left(0<s_{n}<1\right) \\
& \leq(2 \pi e)^{-1 / 2} \max \left(\left|s_{n}-1\right|,\left|s_{n}-1\right| / s_{n}\right) \\
& \leq C_{1} \max \left(\left|s_{n}-1\right|,\left|s_{n}-1\right| / s_{n}\right) \cdot\left(s_{n}+1\right) \quad\left(\text { note that } s_{n} \rightarrow 1\right) \\
& \leq C_{2}\left|s_{n}^{2}-1\right|=O\left(n^{-1 / 6}\right),
\end{aligned}
$$

and by (2.22),

$$
D_{2}=\sup _{-\infty<t<\infty}\left|P\left(\frac{H_{n}}{s_{n}} \leq \frac{t}{s_{n}}\right)-\Phi\left(\frac{t}{s_{n}}\right)\right|=O\left(n^{-1 / 6}\right) .
$$

Therefore, it follows from (2.16), (2.17), (2.19), (2.23), (2.24) and (2.25) that

$$
\sup _{-\infty<t<\infty}\left|P\left(S_{n}^{\prime} \leq t\right)-\Phi(t)\right|=O\left(n^{-1 / 9}\right)+O\left(n^{-1 / 6}\right)=O\left(n^{-1 / 9}\right) .
$$

Finally, by (2.7), (2.10), (2.11) and (2.26), (2.1) holds true. $\square$

Lemma 2.2 Let $\left\{X_{n}\right\}_{n \geq 1}$ be a second-order stationary NA sequence with common marginal distribution function and $E X_{n}=0,\left|X_{n}\right| \leq d<\infty, n=1,2, \ldots$. We give an assumption such that $\sum_{j=2}^{\infty} j\left|\operatorname{Cov}\left(X_{1}, X_{j}\right)\right|<\infty$. If $\operatorname{Var}\left(X_{1}\right)+2 \sum_{j=2}^{\infty} \operatorname{Cov}\left(X_{1}, X_{j}\right)=\sigma_{1}^{2}>0$, then

$$
\sup _{-\infty<t<\infty}\left|P\left(\frac{\sum_{i=1}^{n} X_{i}}{\sqrt{n} \sigma_{1}} \leq t\right)-\Phi(t)\right|=O\left(n^{-1 / 9}\right), \quad n \rightarrow \infty .
$$

Proof Define $\sigma_{n}^{2}=\operatorname{Var}\left(\sum_{i=1}^{n} X_{i}\right), \sigma^{2}\left(n, \sigma_{1}^{2}\right)=n \sigma_{1}^{2}$ and $\gamma(k)=\operatorname{Cov}\left(X_{i+k}, X_{i}\right)$ for $k=0,1$, $2, \ldots$. For the second-order stationarity process $\left\{X_{n}\right\}_{n \geq 1}$ with common marginal 
distribution function, it can be found by the condition $\sum_{j=1}^{\infty} j|\gamma(j)|<\infty$ that

$$
\begin{aligned}
\left|\sigma_{n}^{2}-\sigma^{2}\left(n, \sigma_{1}^{2}\right)\right| & =\left|n \gamma(0)+2 n \sum_{j=1}^{n-1}\left(1-\frac{j}{n}\right) \gamma(j)-n \gamma(0)-2 n \sum_{j=1}^{\infty} \gamma(j)\right| \\
& =\left|2 n \sum_{j=1}^{n-1} \frac{j}{n} \gamma(j)-2 n \sum_{j=n}^{\infty} \gamma(j)\right| \\
& \leq 2 \sum_{j=1}^{\infty} j|\gamma(j)|+2 n \sum_{j=n}^{\infty}|\gamma(j)| \\
& \leq 4 \sum_{j=1}^{\infty} j|\gamma(j)|=O(1) .
\end{aligned}
$$

On the other hand,

$$
\begin{gathered}
\sup _{-\infty<t<\infty}\left|P\left(\frac{\sum_{i=1}^{n} X_{i}}{\sigma\left(n, \sigma_{1}^{2}\right)} \leq t\right)-\Phi(t)\right| \\
\leq \sup _{-\infty<t<\infty}\left|P\left(\frac{\sum_{i=1}^{n} X_{i}}{\sigma_{n}} \leq \frac{\sigma\left(n, \sigma_{1}^{2}\right)}{\sigma_{n}} t\right)-\Phi\left(\frac{\sigma\left(n, \sigma_{1}^{2}\right)}{\sigma_{n}} t\right)\right| \\
+\sup _{-\infty<t<\infty}\left|\Phi\left(\frac{\sigma\left(n, \sigma_{1}^{2}\right)}{\sigma_{n}} t\right)-\Phi(t)\right| \\
:=D_{1}+D_{2} .
\end{gathered}
$$

Obviously, if $b_{n} \rightarrow \infty$ as $n \rightarrow \infty$, then it follows from $\sum_{j=2}^{\infty} j\left|\operatorname{Cov}\left(X_{1}, X_{j}\right)\right|<\infty$ that

$$
\sum_{j=b_{n}}^{\infty}\left|\operatorname{Cov}\left(X_{1}, X_{j}\right)\right| \leq \frac{1}{b_{n}} \sum_{j=b_{n}}^{\infty} j\left|\operatorname{Cov}\left(X_{1}, X_{j}\right)\right|=o\left(b_{n}^{-1}\right) .
$$

(2.28) and the fact $\sigma^{2}\left(n, \sigma_{1}^{2}\right)=n \sigma_{1}^{2} \rightarrow \infty$ yield that $\lim _{n \rightarrow \infty} \sigma_{n}^{2} / \sigma^{2}\left(n, \sigma_{1}^{2}\right)=1$. Thus, by Lemma 2.1,

$$
D_{1}=O\left(n^{-1 / 9}\right) .
$$

By (2.28) again and similar to the proof of (2.24), it follows

$$
D_{2} \leq C\left|\frac{\sigma_{n}^{2}}{\sigma^{2}\left(n, \sigma_{1}^{2}\right)}-1\right|=\frac{C}{\sigma^{2}\left(n, \sigma_{1}^{2}\right)}\left|\sigma_{n}^{2}-\sigma^{2}\left(n, \sigma_{1}^{2}\right)\right|=O\left(n^{-1}\right) .
$$

Finally, by (2.29), (2.30) and (2.31), (2.27) holds true.

Remark 2.1 Under the conditions of Lemma 2.2, we have (27). Furthermore, by the proof of Lemma 2.2, we can obtain that

$$
\sup _{-\infty<t<\infty}\left|P\left(\frac{\sum_{i=1}^{n} X_{i}}{\sqrt{n} \sigma_{1}} \leq t\right)-\Phi(t)\right| \leq C\left(\sigma_{1}^{2}\right) n^{-1 / 9}, \quad n \rightarrow \infty,
$$

where $C\left(\sigma_{1}^{2}\right)$ is a positive constant depending only on $\sigma_{1}^{2}$.

\section{Proof of the main result}

Proof of Theorem 1.1 The proof is inspired by the proofs of Theorem A and Theorem $\mathrm{C}$ of Serfling [[10], pp. 77-84]. Denote $A=\sigma\left(\xi_{p}\right) / f\left(\xi_{p}\right)$ and

$$
G_{n}(t)=P\left(n^{1 / 2}\left(\xi_{p, n}-\xi_{p}\right) / A \leq t\right) .
$$


Let $L_{n}=(\log n \log \log n)^{1 / 2}$, we have

$$
\begin{aligned}
\sup _{|t|>L_{n}}\left|G_{n}(t)-\Phi(t)\right| & =\max \left\{\sup _{t<-L_{n}}\left|G_{n}(t)-\Phi(t)\right|, \sup _{t>L_{n}}\left|G_{n}(t)-\Phi(t)\right|\right\} \\
& \leq \max \left\{G_{n}\left(-L_{n}\right)+\Phi\left(-L_{n}\right), 1-G_{n}\left(L_{n}\right)+1-\Phi\left(L_{n}\right)\right\} \\
& \leq G_{n}\left(-L_{n}\right)+1-G_{n}\left(L_{n}\right)+1-\Phi\left(L_{n}\right) \\
& \leq P\left(\left|\xi_{p, n}-\xi_{p}\right| \geq A L_{n} n^{-1 / 2}\right)+1-\Phi\left(L_{n}\right) .
\end{aligned}
$$

Since $1-\Phi(x) \leq \frac{(2 \pi)^{-1 / 2}}{x} e^{-x^{2} / 2}, x>0$ it follows

$$
1-\Phi\left(L_{n}\right) \leq \frac{(2 \pi)^{-1 / 2}}{L_{n}} e^{-\log n \log \log n / 2}=O\left(n^{-1}\right) .
$$

Let $\varepsilon_{n}=\left(A-\varepsilon_{0}\right)(\log n \log \log n)^{1 / 2} n^{-1 / 2}$, where $0<\varepsilon_{0}<A$. Seeing that

$$
P\left(\left|\xi_{p, n}-\xi_{p}\right| \geq A(\log n \log \log n)^{1 / 2} n^{-1 / 2}\right) \leq P\left(\left|\xi_{p, n}-\xi_{p}\right|>\varepsilon_{n}\right)
$$

and

$$
P\left(\left|\xi_{p, n}-\xi_{p}\right|>\varepsilon_{n}\right)=P\left(\xi_{p, n}>\xi_{p}+\varepsilon_{n}\right)+P\left(\xi_{p, n}<\xi_{p}-\varepsilon_{n}\right),
$$

by Lemma A.4 (iii), we obtain

$$
\begin{aligned}
P\left(\xi_{p, n}>\xi_{p}+\varepsilon_{n}\right) & =P\left(p>F_{n}\left(\xi_{p}+\varepsilon_{n}\right)\right)=P\left(1-F_{n}\left(\xi_{p}+\varepsilon_{n}\right)>1-p\right) \\
& =P\left(\sum_{i=1}^{n} I\left(X_{i}>\xi_{p}+\varepsilon_{n}\right)>n(1-p)\right) \\
& =P\left(\sum_{i=1}^{n}\left(V_{i}-E V_{i}\right)>n \delta_{n 1}\right),
\end{aligned}
$$

where $V_{i}=I\left(X_{i}>\xi_{p}+\xi_{n}\right)$ and $\delta_{n 1}=F\left(\xi_{p}+\varepsilon_{n}\right)-p$. Likewise,

$$
P\left(\xi_{p, n}<\xi_{p}-\varepsilon_{n}\right) \leq P\left(p \leq F_{n}\left(\xi_{p}-\varepsilon_{n}\right)\right)=P\left(\sum_{i=1}^{n}\left(W_{i}-E W_{i}\right) \geq n \delta_{n 2}\right),
$$

where $W_{i}=I\left(X_{i}>\xi_{p}-\xi_{\mathrm{n}}\right)$ and $\delta_{n 2}=p-F\left(\xi_{p}-\varepsilon_{n}\right)$. It is easy to see that $\left\{V_{i}-E V_{i}\right\}$ $1 \leq i \leq n$. and $\left\{W_{i}-E V_{i}\right\}_{1 \leq i \leq n}$ are still NA sequences. Obviously, $\left|V_{i}-E V_{i}\right| \leq 1$, $\sum_{i=1}^{n} E\left(V_{i}-E V_{i}\right)^{2} \leq n,\left|W_{i}-E W_{i}\right| \leq 1, \sum_{i=1}^{n} E\left(W_{i}-E W_{i}\right)^{2} \leq n$. By Lemma A.2, we have that

$$
\begin{aligned}
& P\left(\xi_{p, n}>\xi_{p}+\varepsilon_{n}\right) \leq 2 \exp \left\{-\frac{n \delta_{n 1}^{2}}{2\left(2+\delta_{n 1}\right)}\right\}, \\
& P\left(\xi_{p, n}<\xi_{p}-\varepsilon_{n}\right) \leq 2 \exp \left\{-\frac{n \delta_{n 2}^{2}}{2\left(2+\delta_{n 2}\right)}\right\} .
\end{aligned}
$$

Consequently,

$$
P\left(\left|\xi_{p, n}-\xi_{p}\right|>\varepsilon_{n}\right) \leq 4 \exp \left\{-\frac{n\left[\min \left(\delta_{n 1}, \delta_{n 2}\right)\right]^{2}}{2\left(2+\max \left(\delta_{n 1}, \delta_{n 2}\right)\right)}\right\} .
$$

Since $F(x)$ is continuous at $\xi_{p}$ with $F^{\prime}\left(\xi_{p}\right)>0, \xi_{p}$ is the unique solution of $F(x-) \leq p$ $\leq F(x)$ and $F\left(\xi_{p}\right)=p$. By the assumption on $f(x)$ and Taylor's expansion,

$$
\begin{aligned}
& F\left(\xi_{p}+\varepsilon_{n}\right)-p=F\left(\xi_{p}+\varepsilon_{n}\right)-F\left(\xi_{p}\right)=f\left(\xi_{p}\right) \varepsilon_{n}+o\left(\varepsilon_{n}\right), \\
& p-F\left(\xi_{p}-\varepsilon_{n}\right)=F\left(\xi_{p}\right)-F\left(\xi_{p}-\varepsilon_{n}\right)=f\left(\xi_{p}\right) \varepsilon_{n}+o\left(\varepsilon_{n}\right) .
\end{aligned}
$$


Therefore, we can get that for $n$ large enough,

$$
\begin{aligned}
\frac{f\left(\xi_{p}\right) \varepsilon_{n}}{2} & =\frac{f\left(\xi_{p}\right)\left(A-\varepsilon_{0}\right)(\log n \log \log n)^{1 / 2}}{2 n^{1 / 2}} \leq F\left(\xi_{p}+\varepsilon_{n}\right)-p, \\
\frac{f\left(\xi_{p}\right) \varepsilon_{n}}{2} & =\frac{f\left(\xi_{p}\right)\left(A-\varepsilon_{0}\right)(\log n \log \log n)^{1 / 2}}{2 n^{1 / 2}} \leq p-F\left(\xi_{p}-\varepsilon_{n}\right) .
\end{aligned}
$$

Note that $\max \left(\delta_{n 1}, \delta_{n 2}\right) \rightarrow 0$. as $n \rightarrow \infty$. So with (3), for $n$ large enough,

$$
P\left(\left|\xi_{p, n}-\xi_{p}\right|>\varepsilon_{n}\right) \leq 4 \exp \left\{-\frac{f^{2}\left(\xi_{p}\right)\left(A-\varepsilon_{0}\right)^{2} \log n \log \log n}{8\left(2+\max \left(\delta_{n 1}, \delta_{n 2}\right)\right)}\right\}=O\left(n^{-1}\right) .
$$

Next, we define

$$
\sigma^{2}(n, t)=\operatorname{Var}\left(Z_{1}\right)+2 \sum_{j=2}^{\infty} \operatorname{Cov}\left(Z_{1}, Z_{j}\right),
$$

where $Z_{i}=I\left[X_{i} \leq \xi_{p}+t A n^{-1 / 2}\right]-E I\left[X_{i} \leq \xi_{p}+t A n^{-1 / 2}\right]$. Seeing that

$$
\sigma^{2}\left(\xi_{p}\right)=\operatorname{Var}\left[I\left(X_{1} \leq \xi_{p}\right)\right]+2 \sum_{j=2}^{\infty} \operatorname{Cov}\left[I\left(X_{1} \leq \xi_{p}\right), I\left(X_{j} \leq \xi_{p}\right)\right],
$$

we will estimate the convergence rate of $\left|\sigma^{2}(n, t)-\sigma^{2}\left(\xi_{p}\right)\right|$. By the condition (1.1), we can see that $\sigma^{2}\left(\xi_{p}\right)<\infty$. Since that $F$ possesses a positive continuous density $f$ and a bounded second derivative $F^{\prime}$, for $|t| \leq L_{n}=(\log n \log \log n)^{1 / 2}$, we will obtain by Taylor's expansion that

$$
\begin{aligned}
& \left|\operatorname{Var}\left(Z_{1}\right)-\operatorname{Var}\left[I\left(X_{1} \leq \xi_{p}\right)\right]\right| \\
= & \left|\operatorname{Var}\left[I\left(X_{1} \leq \xi_{p}+t A n^{-1 / 2}\right)\right]-\operatorname{Var}\left[I\left(X_{1} \leq \xi_{p}\right)\right]\right| \\
= & \left|F\left(\xi_{p}+t A n^{-1 / 2}\right)-F\left(\xi_{p}\right)+\left[F^{2}\left(\xi_{p}\right)-F^{2}\left(\xi_{p}+t A n^{-1 / 2}\right)\right]\right| \\
\leq & f\left(\xi_{p}\right) \cdot|t| A n^{-1 / 2}+o\left(|t| A n^{-1 / 2}\right) \\
& +\left|F\left(\xi_{p}\right)+F\left(\xi_{p}+t A n^{-1 / 2}\right)\right| \cdot\left[f\left(\xi_{p}\right) \cdot|t| A n^{-1 / 2}+o\left(|t| A n^{-1 / 2}\right)\right] \\
= & O\left((\log n \log \log n)^{1 / 2} n^{-1 / 2}\right) .
\end{aligned}
$$

Similarly, for $j \geq 2$ and $|\mathrm{t}| \leq L_{n}$,

$$
\begin{aligned}
& \left|E\left[I\left(X_{1} \leq \xi_{p}+t A n^{-1 / 2}\right) I\left(X_{j} \leq \xi_{p}+t A n^{-1 / 2}\right)\right]-E\left[I\left(X_{1} \leq \xi_{p}+t A n^{-1 / 2}\right) I\left(X_{j} \leq \xi_{p}\right)\right]\right| \\
\leq & E\left|I\left(X_{j} \leq \xi_{p}+t A n^{-1 / 2}\right)-I\left(X_{j} \leq \xi_{p}\right)\right| \\
= & {\left[F\left(\xi_{p}+t A n^{-1 / 2}\right)-F\left(\xi_{p}\right)\right] I(t \geq 0)+\left[F\left(\xi_{p}\right)-F\left(\xi_{p}+t A n^{-1 / 2}\right)\right] I(t<0) } \\
= & O\left((\log n \log \log n)^{1 / 2} n^{-1 / 2}\right),
\end{aligned}
$$

Therefore, by a similar argument, for $j \geq 2$ and $|\mathrm{t}| \leq L_{n}$,

$$
\begin{aligned}
& \left|\operatorname{Cov}\left(Z_{1}, Z_{j}\right)-\operatorname{Cov}\left[I\left(X_{1} \leq \xi_{p}\right), I\left(X_{j} \leq \xi_{p}\right)\right]\right| \\
\leq & \left|E\left[I\left(X_{1} \leq \xi_{p}+t A n^{-1 / 2}\right) I\left(X_{j} \leq \xi_{p}+t A n^{-1 / 2}\right)\right]-E\left[I\left(X_{1} \leq \xi_{p}\right) I\left(X_{j} \leq \xi_{p}\right)\right]\right| \\
& +\left|E\left[I\left(X_{1} \leq \xi_{p}+t A n^{-1 / 2}\right)\right] E\left[I\left(X_{j} \leq \xi_{p}+t A n^{-1 / 2}\right)\right]-E\left[I\left(X_{1} \leq \xi_{p}\right)\right] E\left[I\left(X_{j} \leq \xi_{p}\right)\right]\right| \\
\leq & \mid E\left[I\left(X_{1} \leq \xi_{p}+t A n^{-1 / 2}\right) I\left(X_{j} \leq \xi_{p}+t A n^{-1 / 2}\right)\right] \\
& -E\left[I\left(X_{1} \leq \xi_{p}+t A n^{-1 / 2}\right) I\left(X_{j} \leq \xi_{p}\right)\right] \mid \\
& +\left|E\left[I\left(X_{1} \leq \xi_{p}+t A n^{-1 / 2}\right) I\left(X_{j} \leq \xi_{p}\right)\right]-E\left[I\left(X_{1} \leq \xi_{p}\right) I\left(X_{j} \leq \xi_{p}\right)\right]\right| \\
& +\mid E\left[I\left(X_{1} \leq \xi_{p}+t A n^{-1 / 2}\right)\right] E\left[I\left(X_{j} \leq \xi_{p}+t A n^{-1 / 2}\right)\right] \\
& -E\left[I\left(X_{1} \leq \xi_{p}+t A n^{-1 / 2}\right)\right] E\left[I\left(X_{j} \leq \xi_{p}\right)\right] \mid \\
& +\left|E\left[I\left(X_{1} \leq \xi_{p}+t A n^{-1 / 2}\right)\right] E\left[I\left(X_{j} \leq \xi_{p}\right)\right]-E\left[I\left(X_{1} \leq \xi_{p}\right)\right] E\left[I\left(X_{j} \leq \xi_{p}\right)\right]\right| \\
= & O\left((\log n \log \log n)^{1 / 2} n^{-1 / 2}\right) .
\end{aligned}
$$


Consequently, by the conditions (1.1) and (3.5), (3.6), for $|t| \leq L_{n}$,

$$
\begin{aligned}
& \left|\sigma^{2}(n, t)-\sigma^{2}\left(\xi_{p}\right)\right| \\
\leq & \left|\operatorname{Var}\left(Z_{1}\right)-\operatorname{Var}\left[I\left(X_{1} \leq \xi_{p}\right)\right]\right| \\
& +2 \sum_{j=2}^{\left[n^{1 / 5}\right]}\left|\operatorname{Cov}\left(Z_{1}, Z_{j}\right)-\operatorname{Cov}\left[I\left(X_{1} \leq \xi_{p}\right), I\left(X_{j} \leq \xi_{p}\right)\right]\right| \\
& +2 \sum_{j=\left[n^{1 / 5}\right]+1}^{\infty}\left|\operatorname{Cov}\left(Z_{1}, Z_{j}\right)\right|+2 \sum_{\left[j=n^{1 / 5}\right]+1}^{\infty}\left|\operatorname{Cov}\left[I\left(X_{1} \leq \xi_{p}\right), I\left(X_{j} \leq \xi_{p}\right)\right]\right| \\
\leq & C_{1}(\log n \log \log n)^{1 / 2} n^{-1 / 2}+C_{2} n^{1 / 5}(\log n \log \log n)^{1 / 2} n^{-1 / 2}+o\left(n^{-1 / 5}\right) \\
= & o\left(n^{-1 / 5}\right) .
\end{aligned}
$$

By Lemma A.4 (iii) again, it has

$$
\begin{aligned}
G_{n}(t) & =P\left(\xi_{p, n} \leq \xi_{p}+t A n^{-1 / 2}\right)=P\left[p \leq F_{n}\left(\xi_{p}+t A n^{-1 / 2}\right)\right] \\
& =P\left[n p \leq \sum_{i=1}^{n} I\left(X_{i} \leq \xi_{p}+t A n^{-1 / 2}\right)\right] \\
& =P\left[\frac{n^{1 / 2}\left(p-F\left(\xi_{p}+t A n^{-1 / 2}\right)\right)}{\sigma(n, t)} \leq \frac{\sum_{i=1}^{n} Z_{i}}{\sqrt{n} \sigma(n, t)}\right] .
\end{aligned}
$$

Thus,

$$
G_{n}(t)=P\left[\frac{\sum_{i=1}^{n} Z_{i}}{\sqrt{n} \sigma(n, t)} \geq-c_{n t}\right]=1-P\left[\frac{\sum_{i=1}^{n} Z_{i}}{\sqrt{n} \sigma(n, t)}<-c_{n t}\right],
$$

where

$$
c_{n t}=\frac{n^{1 / 2}\left(F\left(\xi_{p}+t A n^{-1 / 2}\right)-p\right)}{\sigma(n, t)} .
$$

It is easy to check that

$$
\begin{aligned}
\Phi(t)-G_{n}(t) & =\Phi(t)-1+P\left[\frac{\sum_{i=1}^{n} Z_{i}}{\sqrt{n} \sigma(n, t)}<-c_{n t}\right] \\
& =P\left[\frac{\sum_{i=1}^{n} Z_{i}}{\sqrt{n} \sigma(n, t)}<-c_{n t}\right]-[1-\Phi(t)] \\
& =P\left[\frac{\sum_{i=1}^{n} Z_{i}}{\sqrt{n} \sigma(n, t)}<-c_{n t}\right]-\Phi\left(-c_{n t}\right)+\Phi(t)-\Phi\left(c_{n t}\right) .
\end{aligned}
$$

By (3.7), it has that $\lim \frac{\sigma^{2}(n, t)}{\sigma^{2}\left(\xi_{p}\right)} \rightarrow 1$ as $n \rightarrow \infty$, which implies that $0<\sigma^{2}(n, t)$ for $|\mathrm{t}| \leq$ $L_{n}$ and $n$ large enough. Obviously, $\left\{Z_{i}\right\}$ is a second-order stationary NA sequence. Thus, for a fixed $t,|t| \leq L_{n}$, by the Lemma 2.2, (2.32) in Remark 2.1 and (3.7), it has for $n$ large enough that

$$
\begin{aligned}
& \left|P\left[\frac{\sum_{i=1}^{n} Z_{i}}{\sqrt{n} \sigma(n, t)}<-c_{n t}\right]-\Phi\left(-c_{n t}\right)\right| \\
\leq & \sup _{-\infty<x<\infty}\left|P\left[\frac{\sum_{i=1}^{n} Z_{i}}{\sqrt{n} \sigma(n, t)}<x\right]-\Phi(x)\right| \\
\leq & C\left(\sigma^{2}(n, t)\right) n^{-1 / 9}=C\left(\sigma^{2}\left(\xi_{p}\right)+o\left(n^{-1 / 5}\right)\right) n^{-1 / 9} \\
\leq & C_{1} n^{-1 / 9}
\end{aligned}
$$


where $C_{1}$ does not depend on $t$ for $|t| \leq L_{n}$. Therefore, for $n$ large enough, we have

$$
\sup _{|t| \leq L_{n}}\left|P\left[\frac{\sum_{i=1}^{n} Z_{i}}{\sqrt{n} \sigma(n, t)}<-c_{n t}\right]-\Phi\left(-c_{n t}\right)\right| \leq C_{1} n^{-1 / 9} .
$$

By (3.8) and the inequality above, we can get that for $n$ large enough,

$$
\begin{aligned}
& \sup _{|t| \leq L_{n}}\left|G_{n}(t)-\Phi(t)\right| \\
\leq & \sup _{|t| \leq L_{n}}\left|P\left[\frac{\sum_{i=1}^{n} Z_{i}}{\sqrt{n} \sigma(n, t)}<-c_{n t}\right]-\Phi\left(-c_{n t}\right)\right|+\sup _{|t| \leq L_{n}}\left|\Phi(t)-\Phi\left(c_{n t}\right)\right| \\
\leq & C n^{-1 / 9}+\sup _{|t| \leq L_{n}}\left|\Phi(t)-\Phi\left(c_{n t}\right)\right| .
\end{aligned}
$$

On the other hand,

$$
\begin{aligned}
\sup _{|t| \leq L_{n}}\left|\Phi(t)-\Phi\left(c_{n t}\right)\right| \leq & \sup _{-\infty<t<\infty}\left|\Phi(t)-\Phi\left(\frac{\sigma\left(\xi_{p}\right)}{\sigma(n, t)} t\right)\right| \\
& +\sup _{|t| \leq L_{n}}\left|\Phi\left(\frac{\sigma\left(\xi_{p}\right)}{\sigma(n, t)} t\right)-\Phi\left(c_{n t}\right)\right|:=H_{1}+H_{2} .
\end{aligned}
$$

By (3.7) again and similar to the proof of (2.31), we have

$$
H_{1} \leq C\left|\frac{\sigma^{2}(n, t)}{\sigma^{2}\left(\xi_{p}\right)}-1\right|=C \sigma^{-2}\left(\xi_{p}\right)\left|\sigma^{2}(n, t)-\sigma^{2}\left(\xi_{p}\right)\right|=o\left(n^{-1 / 5}\right) .
$$

By Taylor's expansion again, we obtain that

$$
\begin{aligned}
c_{n t} & =t \cdot \frac{A}{\sigma(n, t)} \cdot \frac{F\left(\xi_{p}+t A n^{-1 / 2}\right)-F\left(\xi_{p}\right)}{t A n^{-1 / 2}} \\
& =t \cdot \frac{A}{\sigma(n, t)} \cdot \frac{F^{\prime}\left(\xi_{p}\right) t A n^{-1 / 2}+\frac{1}{2} F^{\prime \prime}\left(\xi_{p, t}\right)\left(t A n^{-1 / 2}\right)^{2}}{t A n^{-1 / 2}} \\
& =t \frac{\sigma\left(\xi_{p}\right)}{\sigma(n, t)}+t^{2} \frac{A^{2} F^{\prime \prime}\left(\xi_{p, t}\right)}{2 \sigma(n, t)} n^{-1 / 2}
\end{aligned}
$$

where $\xi_{p, t}$ lies between $\xi_{p}$ and $\xi_{p}+A t n^{-1 / 2}$. It is known that [[12], Lemma 5.2],

$$
\sup _{x}|\Phi(x+q)-\Phi(x)| \leq|q| \cdot(2 \pi)^{-1 / 2}, \quad \text { for every } q .
$$

Therefore, by (3.12), (3.13) and the condition that $F^{\prime}$ is bounded in a neighborhood of $\xi_{p}$, we get for $n$ large enough that

$$
H_{2}=\sup _{|t| \leq L_{n}}\left|\Phi\left(\frac{\sigma\left(\xi_{p}\right)}{\sigma(n, t)} t\right)-\Phi\left(c_{n t}\right)\right| \leq C L_{n}^{2} n^{-1 / 2}=O\left(\log n \cdot \log \log n \cdot n^{-1 / 2}\right),
$$

since $\sigma^{2}\left(\xi_{p}\right)<\infty$ and $\lim _{n \rightarrow \infty} \frac{\sigma^{2}(n, t)}{\sigma^{2}\left(\xi_{p}\right)}=1$ for $|t| \leq L_{n}$. Therefore, it follows from (3.9), (3.10), (3.11) and (3.14) that

$$
\sup _{|t| \leq L_{n}}\left|G_{n}(t)-\Phi(t)\right|=O\left(n^{-1 / 9}\right) .
$$

Finally, the desired result (1.3) follows from (3.1), (3.2), (3.4) and (3.15) immediately. 


\section{Appendix}

Lemma A.1 [[22], Theorem 1] Let $\left\{X_{n}\right\}_{n \geq 1}$ be NA random variables, $E X_{i}=0, E\left|X_{i}\right|^{p}<$ $\infty$, where $i=1,2, \ldots, n$ and $p \geq 2$. Then, there exists some constant $c_{p}$ depending only on p such that

$$
E\left|\sum_{i=1}^{n} X_{i}\right|^{p} \leq c_{p}\left\{\sum_{i=1}^{n} E\left|X_{i}\right|^{p}+\left(\sum_{i=1}^{n} E X_{i}^{2}\right)^{p / 2}\right\} .
$$

Lemma A.2 [[16], Lemma 3.5] Let $\left\{X_{n}\right\}_{n \geq 1}$ be a NA sequence with $E X_{i}=0,\left|X_{i}\right| \leq b$, a.s. $i=1,2, \ldots$, Denote $\Delta_{n}=\sum_{i=1}^{n} E X_{i}^{2}$. Then for $\forall \varepsilon>0$,

$$
P\left(\left|\sum_{i=1}^{n} X_{i}\right|>\varepsilon\right) \leq 2 \exp \left\{-\frac{\varepsilon^{2}}{2\left(2 \Delta_{n}+b \varepsilon\right)}\right\} .
$$

Lemma A.3 [[23], Lemma 2] Let $\times$ and $Y$ be random variables, then for any $a>0$,

$$
\sup _{t}|P(X+Y \leq t)-\Phi(t)| \leq \sup _{t}|P(X \leq t)-\Phi(t)|+\frac{a}{\sqrt{2 \pi}}+P(|Y|>a) .
$$

Lemma A.4 [[10], Lemma 1.1.4] Let $F(x)$ be a right-continuous distribution function. The inverse function $F^{-1}(t), 0<t<1$, is nondecreasing and left-continuous, and satisfies

(i) $F^{-1}(F(x)) \leq x,-\infty<x<\infty$;

(ii) $F\left(F^{-1}(t)\right) \geq t, 0<t<1$;

(ii) $F(x) \geq t$ if and only if $x \geq F^{-1}(t)$.

\section{Acknowledgements}

The authors are most grateful to the Editor Charles E. Chidume and an anonymous referee for the careful reading of the manuscript and valuable suggestions which helped in significantly improving an earlier version of this paper. Supported by the NNSF of China (11171001, 61075009), HSSPF of the Ministry of Education of China (10YJA910005), Provincial Natural Science Research Project of Anhui Colleges (KJ2010A005), Talents youth Fund of Anhui Province Universities (2010SQRL016ZD) and Youth Science Research Fund of Anhui University (2009QN011A).

\section{Author details}

${ }^{1}$ School of Mathematical Science, Anhui University Hefei 230039, PR China ${ }^{2}$ Department of Statistics and Finance University of Science and Technology of China Hefei 230026, PR China

\section{Authors' contributions}

Under some weak conditions, the Berry-Esséen bound of the sample quantiles for NA sequence is presented as $O$ ( $n^{-}$ ${ }^{1 / 9}$ ). All authors read and approved the final manuscript.

\section{Competing interests}

The authors declare that they have no competing interests.

Received: 4 January 2011 Accepted: 10 October 2011 Published: 10 October 2011

\section{References}

1. Bahadur, RR: A note on quantiles in large samples. Ann. Math. Stat. 37, 577-580 (1966). doi:10.1214/aoms/1177699450

2. Sen, PK: On Bahadur representation of sample quantile for sequences of $\phi$-mixing random variables. J. Multivar. Anal. 2, 77-95 (1972). doi:10.1016/0047-259X(72)90011-5

3. Babu, GJ, Singh, K: On deviations between empirical and quantile processes for mixing random variables. J. Multivar. Anal. 8, 532-549 (1978). doi:10.1016/0047-259X(78)90031-3

4. Yoshihara, K: The Bahadur representation of sample quantile for sequences of strongly mixing random variables. Stat. Probab. Lett. 24, 299-304 (1995). doi:10.1016/0167-7152(94)00187-D

5. Sun, SX: The Bahadur representation for sample quantiles under weak dependence. Stat. Probab. Lett. 76, 1238-1244 (2006). doi:10.1016/j.spl.2005.12.021

6. Ling, NX: The Bahadur representation for sample quantiles under negatively associated sequence. Stat. Probab. Lett. 78, 2660-2663 (2008). doi:10.1016/j.spl.2008.03.026

7. $\mathrm{Li}, \mathrm{XQ}$, Yang, WZ, Hu, SH, Wang, XJ: The Bahadur representation for sample quantile under NOD sequence. J. Nonparametric Stat. 23(1), 59-65 (2011). doi:10.1080/10485252.2010.486033

8. Xing, GD, Yang, SC: A remark on the Bahadur representation of sample quantiles for negatively associated sequences. J. Korean Stat. Soc. 40(3), 277-280 (2011). doi:10.1016/j.jkss.2010.10.006 
9. Wang, XJ, Hu, SH, Yang, WZ: The Bahadur representation for sample quantiles under strongly mixing sequence. J. Stat. Plan. Inf. 141, 655-662 (2010)

10. Serfling, RJ: Approximation Theorems of Mathematical Statistics. Wiley, New York (1980)

11. Shiryaev, AN: Probability, 2nd edn. Springer, New York (1989)

12. Petrov, W: Limit Theorems of Probability Theory: Sequences of Independent Random Variables. Oxford University Press Inc., New York (1995)

13. Hall, P: Martingale Limit Theory and Its Application. Academic Press, Inc., New York (1980)

14. Cai, ZW, Roussas, GG: Smooth estimate of quantiles under association. Stat. Probab. Lett. 36, 275-287 (1997). doi:10.1016/S0167-7152(97)00074-6

15. Cai, ZW, Roussas, GG: Berry-Esséen bounds for smooth estimator of a distribution function under association. J. Nonparametric Stat. 11, 79-106 (1999). doi:10.1080/10485259908832776

16. Yang, SC: Uniformly asymptotic normality of the regression weighted estimator for negatively associated samples. Stat. Probab. Lett. 62, 101-110 (2003)

17. Wang, JF, Zhang, LX: A Berry-Esséen theorem for weakly negatively dependent random variables and its applications. Acta Math. Hung. 110(4):293-308 (2006). doi:10.1007/s10474-006-0024-x

18. Liang, HY, Baek, J: Berry-Esséen bounds for density estimates under NA assumption. Metrika. 68, 305-322 (2008). doi:10.1007/s00184-007-0159-y

19. Liang, HY, De Uña-Álvarez, J: A Berry-Esséen type bound in kernel density estimation for strong mixing censored samples. J. Multivar. Anal. 100, 1219-1231 (2009). doi:10.1016/j.jmva.2008.11.001

20. Lahiri, SN, Sun, S: A Berry-Esséen theorem for samples quantiles under weak dependent. Ann. Appl. Probab. 19(1), 108-126 (2009). doi:10.1214/08-AAP533

21. Chaubey, YP, Dewan, I, Li, J: Smooth estimation of survival and density functions for a stationary associated process using Poisson weights. Stat. Probab. Lett. 81, 267-276 (2011). doi:10.1016/j.spl.2010.10.010

22. Su, C, Zhao, LC, Wang, YB: Moment inequalities and weak convergence for negatively associated sequences. Sci. China A. 40, 172-182 (1997). doi:10.1007/BF02874436

23. Chang, MN, Rao, PV: Berry-Esséen bound for the Kaplan-Meier estimator. Commun. Stat. Theory Methods. 18(12), 4647-4664 (1989). doi:10.1080/03610928908830180

doi:10.1186/1029-242X-2011-83

Cite this article as: Yang et al:: Berry-Esséen bound of sample quantiles for negatively associated sequence.

Journal of Inequalities and Applications 2011 2011:83.

\section{Submit your manuscript to a SpringerOpen ${ }^{\circ}$ journal and benefit from:}

- Convenient online submission

- Rigorous peer review

- Immediate publication on acceptance

- Open access: articles freely available online

- High visibility within the field

- Retaining the copyright to your article

Submit your next manuscript at $\gg$ springeropen.com 